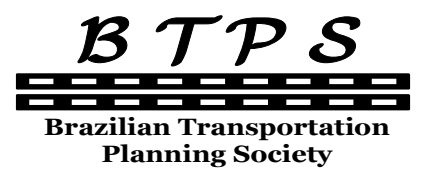
Journal of Transport Literature
Vol. 8, n. 4, pp. 252-278, Oct. 2014
Research Directory

JTL|RELIT

www.journal-of-transport-literature.org ISSN 2238-1031

\title{
As potencialidades do mercado da aviação chinesa para o Aeroporto Internacional de Lisboa: análise de modelagem empírica
}

\author{
[The potentialities of Chinese airline market for Lisbon International Airport: empirical modeling analysis] \\ José Manuel Vicente*, Andreia Dionísio, Manuela Oliveira, Cátia Simão \\ University of Évora - Portugal, University of Évora - Portugal, \\ University of Évora - Portugal, Universidade Lusófona de Humanidades e Tecnologias - Portugal
}

Submitted 27 Jul 2013; received in revised form 15 Mar 2014; accepted 7 Jun 2014

\begin{abstract}
Resumo
A elaboração do presente artigo tem como principal objectivo analisar as potencialidades do mercado da aviação chinesa que se encontra em franca expansão no âmbito do desenvolvimento do aeroporto internacional de Lisboa através de uma análise empírica baseada em modelos com dados de painel aplicados ao contexto dos transportes, nomeadamente o sector do transporte aéreo. Para além do objectivo apresentado anteriormente foi analisado o conceito de nível de circuito e, pela primeira vez, aplicado ao contexto português de forma a verificar o seu carácter benéfico para o desenvolvimento e adequado aproveitamento das condições geográficas da principal infra-estrutura aeronáutica portuguesa. Utilizou-se dois modelos gravitacionais com dados em painel. Estes modelos econométricos permitem o estudo da atractividade entre a origem e o destino no transporte aéreo. Neste trabalho encontra-se uma breve reflexão e introdução de um conceito ainda pouco explorado na bibliografia mundial e nomeadamente no sector do transporte aéreo, o nível de circuito. A aplicabilidade deste conceito no presente trabalho de investigação permitiu aferir a qualidade geográfica de um conjunto de infra-estruturas aeroportuárias que servem de hub em determinadas rotas, concluindo-se que quanto menor for este parâmetro melhor a qualidade de serviço deste hub, sendo como tal possível afirmar que nenhum outro desvio de rota permite transportar os passageiros num período de tempo mais reduzido.
\end{abstract}

Palavras-Chave: Lisboa, modelo gravitacional, modelo de dados em painel, nível de circuito, transporte aéreo.

\begin{abstract}
The development of this paper aims to analyze the potential of the Chinese aviation market that is booming in the development of the Lisbon International Airport through empirical analysis based on panel data models applied to the transport context, in particular in the air transport sector. In addition to the previously stated objective was analyzed the concept of circuit level and, for the first time, applied to the national context in order to verify its beneficial nature for the development and appropriate use of geographical conditions of the main Portuguese aviation infrastructure. Two gravity models with panel data were used. These econometric models allow the study of attractiveness between origin and destination in air transport. In this paper there is a brief reflection and introducing of a new concept that is still little explored in the world literature and particularly in the aviation sector, the circuit level. The applicability of this concept in this research work allowed the assessment of the geographical quality of a set of airport infrastructure serving hub on certain routes, concluding that the lower this parameter the best is the quality of service at this hub, and as such possible to say that no other route diversion let them to carry passengers in a shorter period of time.
\end{abstract}

Key words: Lisbon, gravity model, panel data modeling, circuit level, air transport.

*Email: jose.manuel.vicente81@gmail.com.

\section{Recommended Citation}

Vicente, J. M., Dionísio, A., Oliveira, M. and Simão, C. (2014) As potencialidades do mercado da aviação chinesa para o Aeroporto Internacional de Lisboa: análise de modelagem empírica. Journal of Transport Literature, vol. 8, n. 4, pp. 252-278.

- JTL/RELIT is a fully electronic, peer-reviewed, open access, international journal focused on emerging transport markets and published by BPTS - Brazilian Transport Planning Society. Website www.journal-of-transport-literature.org. ISSN 2238-1031.

This paper is downloadable at dx.doi.org/10.1590/2238-1031.jtl.v8n4a10 


\section{Introdução}

$\mathrm{Na}$ actual economia mundial amplamente multifacetada, o sector do transporte aéreo e, consequentemente do turismo, são cada vez mais vistos como duas das principais fontes para a geração de desenvolvimento socioeconómico numa determinada região e até mesmo de um determinado país. Por este facto, muitos autores e especialistas das temáticas em causa atribuem frequentemente a estes dois sectores a denominação de "exportador invisível" devido ao seu potencial, por vezes, subvalorizado pelos governos e entidades competentes pelo seu correcto funcionamento e constante evolução.

Por outro lado, vários países têm nestas duas indústrias a principal fonte de captação de recursos externos e de investimentos e, em alguns casos particulares, como por exemplo, em pequenos países subdesenvolvidos, estes sectores representam a única fonte viável para o correspondente desenvolvimento.

Apesar de Portugal não ser considerado um país subdesenvolvido, ambiciona-se com a conclusão deste trabalho de investigação fornecer pistas quanto à potencialidade do aeroporto internacional de Lisboa com o intuito de criar e/ ou explorar rotas que dependam do mesmo para a ligação entre duas cidades geograficamente distantes e com a impossibilidade da realização de voos directos entre as mesmas, nomeadamente localizadas no mercado chinês. Dessa forma pretende-se avaliar a atractividade da principal infra-estrutura aeroportuária portuguesa, comparativamente a outros aeroportos, ao longo de um período de tempo previamente estipulado e utilizando, para isso, dois modelos matemáticos com características marcadamente pertencentes à metodologia dos modelos gravitacionais com dados de painel, sendo que cada um deles possui características próprias, de forma a interpretar de forma mais adequada os seus resultados. Os modelos que serão definidos para a concretização do estudo serão constituídos por um conjunto de variáveis seleccionadas devido à sua importância e interligação com o sector do transporte aéreo e, consequentemente, do turismo. Ambos os modelos têm como objectivo determinar a influência de cada conjunto de variáveis para a variação positiva ou negativa do fluxo de passageiros, de forma a verificar o impacto das mesmas para o crescimento do sector do transporte aéreo. A criação de modelos distintos teve como objectivo analisar qual deles poderia dar melhor resposta para o que se pretende estudar neste trabalho, verificando-se que a correcta escolha das variáveis promove uma melhor qualidade de resultados.

Nas últimas décadas tem sido bastante visível o crescimento do sector do transporte aéreo e nomeadamente o tráfego aéreo em diversas regiões do Mundo, principalmente aquelas que beneficiaram 
de uma melhor condição financeira e, principalmente, as que possuem uma relevância significativa para o sector do turismo, seja de lazer ou de cariz profissional.

Através da percepção da evolução temporal do volume de tráfego para o aeroporto em estudo, é possível confirmar o seu crescimento acentuado nesta última década, podendo a mesma ser justificada devido não só ao vasto conjunto de atracções turísticas como também pelo desenvolvimento, criação e operação de cada vez mais companhias de baixo custo para esta infra-estrutura aeroportuária, commumente denominadas por companhias LCC (Low Cost Carriers). A implementação deste tipo de voos aumentou não só o número de voos, como também o número de passageiros, permitindo dessa forma acolher outro tipo de público-alvo que até então não teria acesso, de forma tão frequente, aos serviços prestados pelo sector do transporte aéreo (Forsyth, 2005).

Tal como foi referido anteriormente, para Portugal e nomeadamente na região em que se insere o aeroporto internacional de Lisboa, a importância turística desta região e a adequada posição geográfica relativamente a outros pontos no Mundo, faz com que o interesse turístico por parte de turistas estrangeiros tenha sofrido uma evolução significativa.

Por outro lado, a comparação entre o custo de vida em Portugal e em outros países, faz com que a taxa de crescimento do sector do turismo seja crescente ao longo dos últimos anos, apesar da crise económica existente nos últimos anos. De acordo com esta evidência é possível afirmar que o sector do turismo, nomeadamente o sector hoteleiro na região do aeroporto internacional de Lisboa, tem sofrido uma evolução ao longo da última década através do crescimento praticamente constante do número de dormidas nos diversos tipos de estabelecimentos hoteleiros, nomeadamente, hotéis, hotéisapartamentos, apartamentos turísticos, aldeamentos turísticos, móteis, pousadas, estalagens e pensões.

Devido ao constante crescimento do mercado aeronáutico e nomeadamente o crescimento acentuado em algumas regiões do Mundo, como por exemplo no mercado da aviação chinesa, o número de rotas de voos entre os aeroportos é cada vez maior e com elevada tendência e potencialidade para ligar as principais cidades mundiais a qualquer ponto, independentemente da distância que as separa.

Apesar de não existirem voos directos entre diversos pares de cidades, a utilização de voos em codeshare dentro da aliança a que pertencem as companhias de transporte aéreo faz com que essa lacuna seja facilmente ultrapassada. Para além da estratégia apresentada anteriormente, o recurso a hubs internacionais para a realização de escalas permite a minimização da lacuna citada, tal como se poderá analisar ao longo deste trabalho de investigação. 
De forma a cumprir o objectivo a que se propõe a realização deste trabalho de investigação, o mesmo encontra-se dividido em três secções. A Secção 1 pretende fazer uma breve revisão literária à metodologia em que se baseia a criação dos modelos que serão estudados, sendo concluída com a subsecção contendo os dois modelos e a sua consequente apresentação. A Secção 2 contém, em primeiro lugar, os resultados obtidos para cada um dos modelos com recurso ao software econométrico Stata $($ ) (Data Analysis and Statistical Software). Este programa permite obter os resultados de acordo com diferentes métodos de estimação; em segundo lugar será realizada uma comparação entre os resultados obtidos em cada um dos modelos de forma a definir qual deles apresenta resultados de melhor qualidade, tendo em conta o objectivo do estudo. A Secção 3 tem como principal objectivo fornecer um conjunto de estratégias que tragam beneficios para o crescimento do sector do transporte aéreo em Lisboa.

\section{Metodologia}

Apesar de inúmeros investigadores e especialistas reconhecerem a necessidade e a importância da aplicação de abordagens metodológicas para a análise do impacto do sector do transporte aéreo e consequentemente do turismo nas diversas economias mundiais, a verdade é que muitas das conclusões apresentadas na literatura existente se baseiam principalmente em pesquisas teóricas. E, como tal, ainda são raros os estudos empíricos desenvolvidos ao longo de inúmeros países com a mesma metodologia, de forma a comparar e extrapolar conclusões válidas a partir dos resultados obtidos em cada um desses estudos. Sendo assim, a realização deste trabalho de investigação pretende preencher essa lacuna, nomeadamente no contexto português.

A concreta análise da variação do fluxo de passageiros entre um aeroporto de origem e o correspondente de destino encontra-se fortemente dependente de uma elevada complexidade, tendo em conta as diversas abordagens metodológicas para a sua realização e a quantidade, e consequente qualidade, dos resultados obtidos pelas mesmas.

Para a realização destes estudos podem ser utilizados modelos estatísticos, gravitacionais e até mesmo teóricos. Tendo em conta as suas características únicas e aplicabilidades nos diversos tipos de estudos, cada um deles adequa-se de forma diferente a cada problema em análise, devendo a sua escolha ser efectuada de forma cuidada e consciente, não esquecendo o grau de complexidade e o tipo de dados disponibilizados para a sua concretização.

Tendo em conta a grande maioria dos modelos estatísticos e econométricos existentes é possível verificar que até uma certa altura do século passado, todos eles foram estimados através da utilização de séries transversais ou então séries temporais. Com a evolução da complexidade destes mesmos modelos 
foi possível concluir que cada vez mais aplicações não possuíam os seus dados disponíveis para a correcta aplicação da metodologia relativa às séries transversais e/ ou séries temporais, surgindo a necessidade da utilização de uma metodologia mais eficaz (Washington et al., 2003).

Apesar de o estudo ter sido realizado baseando-se no modelo gravitacional, de acordo com as suas características e o facto dos modelos em análise serem constituídos por dados de painel, o mesmo possui então diversas particularidades. Desse modo, a presente secção pretende efectuar uma revisão literária quanto à aplicabilidade dos dois tipos de modelos para estudos no âmbito do transporte aéreo, referindo as suas semelhanças e diferenças sempre que possível.

\subsection{Modelo com dados de painel}

Ao longo de todo o processo de investigação foram analisadas diferentes abordagens metodológicas, nomeadamente, as séries temporais, as séries transversais e os dados de painel. Tendo em conta a análise ponderada entre as vantagens e as desvantagens promovidas por cada uma dessas metodologias e o tipo de dados recolhidos através das inúmeras fontes consultadas, optou-se pela análise dos dados de painel para a concretização do estudo econométrico. Esta metodologia denominada por dados de painel une as vantagens das duas metodologias enunciadas anteriormente, ultrapassando os seus obstáculos e lacunas, permitindo a construção de modelos mais realistas (Wooldridge, 2002 e 2012).

De uma forma geral é possível referir que esta metodologia consiste numa análise estatística de um conjunto de dados obtidos através da observação repetida dos mesmos indivíduos ao longo de um determinado período de tempo para um conjunto de variáveis pré-estipuladas, tendo como base os princípios de construção de um modelo geral de regressão linear em que se pretende estimar o valor esperado para uma determinada variável dependente (Wooldridge, 2002 e 2012). Isto é, através de um modelo $\mathrm{Y}$ definido a partir de um conjunto de variáveis independentes (também denominadas frequentemente por variáveis explicativas ou regressores) X é possível determinar o comportamento do mesmo ao longo do período desejado t. Logo, uma variação em Y estará directamente relacionada com as variações obtidas ao longo das diversas variáveis X (Wooldridge, 2002 e 2012). A especificidade do modelo de dados de painel baseia-se na sua capacidade de efectuar uma análise estatística recorrendo a dados temporais, denominados na literatura como time-series, e seccionais, cross-section, utilizando um só modelo econométrico (Wooldridge, 2002 e 2012).

Dentro das especificações que constituem o modelo de dados de painel é importante fazer referência aos principais métodos de estimação que serão ao longo deste trabalho de investigação analisados, nomeadamente, os efeitos fixos e os efeitos aleatórios (Marques, 2000): 
- Efeitos fixos: É um método de estimação que tem como principal característica o facto de eliminar todos os efeitos que não sofrem qualquer alteração ao longo do período temporal em análise. A aplicação deste método de estimação assume a hipótese de homogeneidade das observações. É importante ainda referir que este método de estimação promove uma melhor qualidade de resultados, comparativamente ao seguinte método de estimação, quando é aplicado a uma amostra relativamente extensa. Esta característica pode ser explicada devido ao facto de que quanto maior a dimensão da amostra em estudo maior será o número de parâmetros que necessitam de ser estimados, aumentando o grau de dificuldade e morosidade para a sua concretização;

- Efeitos aleatórios: Ao contrário do que se verifica através do método de estimação anterior, pressupõe-se o desconhecimento do comportamento de algumas variáveis ao longo do período temporal em análise, assumindo-se dessa forma a heterogeneidade dos indivíduos. Ao contrário do método de estimação anterior, este método tem a capacidade de obter resultados de qualidade independentemente da dimensão da amostra em análise, representando também o modelo que permite uma maior profundidade para obtenção e correspondente interpretação dos resultados da estimação. A qualidade dos resultados obtidos deve-se ao facto deste modelo se caracterizar pela hipótese de não existência de correlação entre os efeitos que são observados na amostra e os não observados, sendo a principal diferença entre este método de estimação e o anterior.

A escolha de um dos dois métodos de estimação definidos anteriormente deverá ser feita de forma cuidadosa e tendo em conta os pressupostos comportamentais observados, como por exemplo, se é visível que a variação dos indivíduos ocorre devido a factores não aleatórios, significa que a escolha do método de estimação deve recair sobre o método de efeitos fixos.

Uma elevada quantidade de autores e estudiosos não só das metodologias econométricas como também do sector do transporte aéreo e do turismo afirmam que a qualidade das infra-estruturas aeroportuárias, assim como a ligação entre as mesmas e os diversos meios de transporte existentes na cidade em causa, representam um importante factor de atractividade por parte dos turistas e este mesmo factor foi analisado através da abordagem metodológica apresentada (Khadaroo et al., 2007). Khadaroo utilizou o modelo de dados de painel com o intuito de analisar a influência de um conjunto de variáveis para o crescimento do tráfego aéreo e respectivo desenvolvimento da Mauritânia num período temporal entre 1978 e 2003. Uma das variáveis com elevada relevância para o estudo desenvolvido foi a qualidade da infra-estrutura aeroportuária e rede de transportes de forma a verificar se a mesma constituía um factor de atractividade para o fluxo de passageiros. De forma a evitar conclusões precipitadas e erradas, foram construídos três modelos de dados de painel consoante a região do Mundo em que o fluxo de passageiros era proveniente, isto é, um modelo para a Europa e América, um modelo para a Ásia e, por fim, um 
modelo para a África. Para cada modelo foram aplicados os dois métodos de estimação, obtendo-se resultados semelhantes para o método de estimação de efeitos fixos e aleatórios. Através dos resultados obtidos foi possível concluir que o fluxo de passageiros proveniente dos continentes europeu e americano representa o grupo de passageiros que procura uma qualidade de infra-estruturas e consequente rede de transportes o mais semelhante possível ao que possui no seu local de origem, sendo também o grupo que efectua a maior quantidade de trocas de capital na ilha. Assim sendo, e tendo em conta o mercado promissor em que a ilha se encontra, é requerido um investimento para melhoramento e desenvolvimento das infra-estruturas aeroportuárias e rede intermodal de forma a aumentar o grau de atractividade com que os turistas analisam este destino (Khadaroo et al., 2007).

As conclusões obtidas no estudo anterior podem ser facilmente explicadas e extrapoladas para o caso português onde ainda não existe uma intermodalidade de forma integral entre o transporte aéreo, ferroviário e rodoviário, dificultando por vezes a deslocação dos turistas entre os diversos pontos turísticos que pretendem visitar durante a sua estadia em Portugal.

A eficiência e acessibilidade das infra-estruturas aeroportuárias comprometem gravemente não só o desenvolvimento do sector do transporte aéreo como também da economia do país em que se insere e estas conclusões foram retiradas através de um estudo efectuado a treze países europeus, com acesso a dados referentes ao período de 1991 a 2010. Por outro lado, é importante distinguir as metrópoles em que se insere cada uma das infra-estruturas aeroportuárias em análise, tendo em conta a maior ou menor afluência de passageiros de forma a verificar que as principais infra-estruturas aeroportuárias internacionais de cada país são aquelas que promovem um maior efeito positivo para a economia nacional, devido a todo o desenvolvimento regional existente ao seu redor (Mukkala et al., 2012).

Num contexto também referente à actividade das infra-estruturas aeroportuárias, o seu franco crescimento nas últimas décadas, apesar de ter trazido melhorias significativas para todos os utilizadores deste meio de transporte deu origem a problemas ambientais que, para serem combatidos, necessitam de um elevado investimento não só financeiro como também social, por parte de todos os intervenientes no processo (Grampella et al., 2013). O trabalho desenvolvido por Grampella baseou-se num conjunto de dados de painel referentes ao período de 1999 a 2008 no contexto das infra-estruturas aeroportuárias italianas com o intuito de analisar a produção de poluição gasosa e sonora nas regiões em que os mesmos se encontram localizados. Através de um modelo econométrico de dados de painel por efeitos fixos foi possível expressar uma relação directamente proporcional entre o aumento da poluição com o crescimento do sector aeronáutico, seja em termos de aumento de tráfego como também desenvolvimento das aeronaves, nomeadamente o aumento do seu peso e da sua capacidade de 
transporte, não só em termos de passageiros e/ou carga como também de combustível (Grampella et al., 2013).

Para além da utilização desta metodologia para a análise da qualidade das infra-estruturas aeroportuárias, a mesma foi implementada num estudo desenvolvido por Whalen com o intuito de analisar e quantificar a importância e correspondentes efeitos dos acordos de operação entre diversas companhias aéreas, denominados por code-share. O principal objectivo levado a cabo por Whalen baseava-se na determinação do efeito deste tipo de acordos para a variação do preço das passagens aéreas e da capacidade de transporte de passageiros entre diversas localizações, baseando a sua análise econométrica em dados referentes a um período de onze anos e limitado geograficamente aos Estados Unidos da América (E.U.A) e ao território europeu (Whalen, 2005). Como resultado deste estudo foi possível afirmar que a existência de acordos entre companhias aéreas, seja através de code-share ou qualquer outro tipo de alianças, tem a tendência a baixar o preço das passagens aéreas, sendo um factor benéfico para os seus clientes e, por outro lado, têm capacidade para transportar um maior volume de passageiros (Whalen, 2005).

A franca expansão deste sector económico deve-se, em grande parte, ao aparecimento das LCC's e, por esse motivo, $\mathrm{Wu}$ desenvolveu um estudo baseado nos modelos de dados de painel e também modelos gravitacionais com o intuito de verificar o efeito da variação e dispersão dos preços das passagens aéreas nomeadamente no período decorrente do crescimento de duas companhias aéreas, Southwest Airlines e JetBlue Airways, quando comparadas com o aparecimento de novas companhias aéreas, tendo baseado a sua análise num conjunto de dados referentes ao período de 1993 a 2009 (Wu, 2012). Verificou-se que as novas companhias aéreas agem de formas distintas tendo em conta o seu principal concorrente, isto é, no caso da nova companhia aérea operar directamente como concorrente da Southwest Airlines, existe uma tendência para que efectue um corte significativo no valor do preço das passagens aéreas de forma a cativar o seu público-alvo, atitude que não se verifica no caso de operar em simultâneo com a JetBlue Airways (Wu, 2012).

Por fim, e não menos importante, a metodologia econométrica aplicada neste trabalho de investigação encontra-se também patente no estudo desenvolvido por Fourie e Santana-Gallego com o intuito de analisar e verificar as condicionantes para o crescimento do turismo no continente africano, baseandose num conjunto de dados referentes ao período de 1995 a 2008 (Fourie e Santana-Gallego, 2011). Para além da especificação óbvia da relação existente entre o sector do transporte aéreo e o turismo, o estudo levou em linha de conta um vasto conjunto de países de origem dos turistas e resultou num conselho primordial para o constante crescimento do turismo, isto é, o cuidado com a política governamental 
exercida no continente com vista a promover o desenvolvimento turístico e económico do território em análise (Fourie e Santana-Gallego, 2011).

\subsection{Modelo gravitacional}

Os modelos gravitacionais pretendem estudar e avaliar a dinâmica da variação do fluxo de passageiros entre o aeroporto de origem e de destino, não esquecendo a relevância previamente assumida do hub utilizado para a transferência de passageiros e/ou carga. Para este tipo de modelos as variáveis independentes e a dependente encontram-se directamente relacionadas com a origem e o destino mas, por outro lado, as mesmas encontram-se indirectamente relacionadas com a distância que separa estas duas localizações. No entanto, vários autores apesar da designação de modelo gravitacional, não incluem a variável distância no modelo, devido na maior parte dos casos pelo seu comportamento constante (Santos Silva e Tenreyro, 2006).

A ideia que deu origem a este modelo surgiu no século XVII através do Princípio de Gravitação Universal formulado por Isaac Newton, em que o mesmo assumia uma relação estabelecida entre dois objectos que se atraíam entre si. Essa força de atracção dependia directamente da massa de ambos mas, em contrapartida dependia de forma indirecta da distância que os separava. Por outro lado, a sua aplicabilidade para as análises econométricas teve uma adaptação relativamente facilitada visto que, a componente física relativa à massa anteriormente enunciada foi substituída pelo vector massa económica, mantendo o restante da relação, sendo ainda importante referir que este tipo de modelos promove resultados razoáveis e interessantes, tendo como base de apoio os desenvolvimentos teóricos ocorridos ao longo das últimas décadas (Piane e Kume, 2000).

A distância que separa a origem do destino nem sempre obtém a atenção necessária mas através de alguns estudos foi possível evidenciar o seu impacto e, nomeadamente, a influência de variáveis socioeconómicas, como por exemplo, o tamanho das cidades e o seu desenvolvimento económico.

Para além da elevada importância da variável respeitante à distância, a inclusão ao longo do tempo, em que se realizou o estudo, de variáveis dummy teve um impacto bastante relevante para o desenvolvimento e melhoria dos modelos gravitacionais, devido à combinação deste modelo com o LSDV. Esta alteração permitiu a análise de factores que até então não podiam ser quantificados, nomeadamente aspectos culturais e geográficos, tal como será utilizado num dos modelos deste trabalho de investigação. Por outro lado, a evolução constante destes modelos e a forte capacidade de descrever a influência espacial entre o conjunto origem/destino motivou a sua utilização ao longo dos anos para estudos em diversas áreas, nomeadamente no sector dos transportes rodoviários, dando origem a uma 
importante ferramenta para planeamento e ajustamento de desenvolvimento de infra-estruturas imprescindíveis (Sem e Smith, 1995).

Apesar de não existirem muitos estudos que contemplam a aplicação desta metodologia para o sector do transporte aéreo e nomeadamente para a análise particular de rotas aéreas, Peace estudou a influência de factores turísticos para a variação do fluxo de passageiros, nomeadamente a localização dos grandes centros turísticos junto de infra-estruturas aeroportuárias internacionais (Peace, 2003). A mesma conclusão obtida pelo autor anterior pode ser extrapolada para o caso dos passageiros que se deslocam em trabalho com o intuito de promoverem negócios entre duas cidades e/ ou países distintos. Adicionalmente, um dos estudos encontrados ao longo do processo de pesquisa evidencia a influência da distância na variação do fluxo de passageiros, principalmente no que diz respeito ao facto de que quanto maior for a distância, maior será o grau de competitividade no sector do transporte (Grosche et al., 2007).

O sucesso e frequência da utilização deste tipo de modelos deve-se, principalmente, à simplicidade da sua construção assim como à facilidade de interpretação dos resultados obtidos pelo mesmo (Bedo e Dentinho, 2007). Apesar de todas características benéficas explicitadas anteriormente, até ao momento a sua implementação através de dados de painel não tem sido conseguida devido à dificuldade para a construção do modelo e também da recolha de dados viáveis para a obtenção favorável de resultados de cada estimativa (Santos Silva e Tenreyro, 2006).

É importante salientar que apesar da dependência espacial que caracteriza qualquer modelo gravitacional, nem sempre esse factor é tido em conta ao longo da sua análise, o que pode dar origem a um vasto conjunto de problemas que nem sempre são de fácil percepção através do processo de interpretação de resultados da estimativa, nomeadamente a autocorrelação espacial. Devido a este mesmo factor, segundo o estudo efectuado por LeSage e Pace (2008), a metodologia em causa é capaz de promover uma importante e útil explicação para a variação dos fluxos de transporte entre origem e destino (LeSage e Pace, 2008).

\subsection{Dados e modelagem}

Apesar da análise em estudo pretender verificar e testar o fluxo anual de passageiros entre os dois pontos apresentados anteriormente, isto é, o aeroporto internacional de Lisboa e o mercado de aviação chinesa, entre o período de 2002 a 2009, surgiu a necessidade de implementar dois modelos distintos para a concretização dos objectivos a que se propunha este trabalho de investigação, o primeiro mais clássico, semelhante ao tradicional gravitacional e o segundo contemplando variáveis da indústria, sócioeconómicas e comportamentais, que serão mais à frente definidas. 
Para a construção dos dois modelos referidos anteriormente foram analisados dados respeitantes a voos originários de três dos principais aeroportos brasileiros, como serão seguidamente apresentados através dos respectivos códigos IATA, com destino à cidade de Hong Kong, na China, sendo que esses voos funcionaram através da utilização dos hubs já existentes:

- $\quad$ GRU = Guarulhos Internacional;

- GIG = Galeão-António Carlos Jobim Internacional;

- $\quad \mathrm{POA}=$ Salgado Filho Internacional.

Antes da apresentação dos dois modelos definidos para a realização deste estudo é importante salientar a importância da quantidade e variedade de variáveis seleccionadas e consequentemente utilizadas para a construção dos mesmos, visto que caso os modelos de regressão possuam uma quantidade elevada ou reduzida de variáveis explicativas, esse facto poderá dar origem a um conjunto de conclusões incorrectas devido ao sobre ou subajustamento do conjunto de observações efectuadas.

Para a correcta aplicação e estudo de cada uma das regressões lineares múltiplas é necessário, em primeiro lugar, definir cada um dos modelos que se pretende estudar tendo em conta a relação existente entre as diversas variáveis independentes e a variável dependente em análise, sendo que essas expressões se encontram apresentadas seguidamente. A Equação 1 é respeitante ao primeiro modelo, designado como modelo 1, enquanto a Equação 2 é respeitante ao segundo modelo em estudo, designado como o modelo 2 , respectivamente.

$$
\text { Modelo } 1 \rightarrow \text { Passageiros = f (PIB, População, Tempo) }
$$

Tendo em conta a informação apresentada nas subsecções anteriores deste trabalho de investigação e com o intuito de complementar a apresentação do primeiro modelo, surge a necessidade de enumerar e caracterizar as diversas variáveis recolhidas ao longo de todo o processo de investigação e consequentemente analisadas para a construção e posterior implementação dos modelos, encontrandose todas as especificações para o primeiro modelo na Tabela 1. 
Tabela 1 - Apresentação e caracterização dos dados utilizados

\begin{tabular}{|c|c|c|c|}
\hline Variável & Coordenada & Caracterização & Fonte \\
\hline PIB & $\begin{array}{c}\mathrm{X}_{1} \\
\text { Variável } \\
\text { independente }\end{array}$ & $\begin{array}{l}\text { Representa a soma do valor } \\
\text { acrescentado bruto por todos os } \\
\text { produtores residentes na economia e } \\
\text { expresso em dólares americanos }(\$) \text {. }\end{array}$ & $\begin{array}{c}\text { World Bank } \\
\text { (www.worldbank.org) }\end{array}$ \\
\hline População & $\begin{array}{c}\mathrm{X}_{2} \\
\text { Variável } \\
\text { independente }\end{array}$ & $\begin{array}{l}\text { Número total de residentes, } \\
\text { independentemente do seu estatuto } \\
\text { legal e/ ou cidadania. }\end{array}$ & $\begin{array}{c}\text { World Bank } \\
\text { (www.worldbank.org) }\end{array}$ \\
\hline Tempo & $\begin{array}{c}\mathrm{X}_{3} \\
\text { Variável } \\
\text { independente }\end{array}$ & $\begin{array}{l}\text { Representa a unidade temporal que } \\
\text { reportam os dados em análise, o ano } \\
\text { civil (indexação do ano civil às várias } \\
\text { variáveis). }\end{array}$ & Elaboração Própria \\
\hline Passageiros & $\begin{array}{c}\text { Y } \\
\text { Variável } \\
\text { dependente }\end{array}$ & $\begin{array}{l}\text { Número de passageiros transportados } \\
\text { anualmente em cada uma das rotas } \\
\text { estudadas. }\end{array}$ & $\begin{array}{l}\text { Sabre Airport Data } \\
\text { Intelligence } \\
\text { (www.airdi.net) }\end{array}$ \\
\hline
\end{tabular}

A Equação 1 distingue-se da Equação 2 devido não só à quantidade como também à variedade das variáveis independentes seleccionadas para a realização do estudo. A implementação de uma maior quantidade de variáveis independentes tem como principal objectivo melhorar a capacidade de previsão do modelo de regressão linear múltipla quando comparado a um modelo de regressão linear simples ou um modelo de regressão linear múltipla com uma menor quantidade de variáveis (Wooldridge, 2012).

Modelo $2 \rightarrow$ Passageiros $=$ f (PIB, População, Preço da Passagem Aérea, Factor

$$
\text { de Negócio, Factor de Turismo, Tempo) }
$$

Tal como foi feito para o primeiro modelo, a apresentação da Tabela 2 tem como objectivo caracterizar as variáveis respeitantes ao segundo modelo. 
Tabela 2 - Apresentação e caracterização dos dados utilizados

\begin{tabular}{|c|c|c|c|}
\hline Variável & Coordenada & Caracterização & Fonte \\
\hline PIB & $\begin{array}{c}\mathrm{X}_{1} \\
\text { Variável } \\
\text { independente }\end{array}$ & $\begin{array}{l}\text { Representa a soma do valor } \\
\text { acrescentado bruto por todos os } \\
\text { produtores residentes na economia e } \\
\text { expresso em dólares americanos }(\$) \text {. }\end{array}$ & $\begin{array}{l}\text { World Bank } \\
\text { (www.worldbank.org) }\end{array}$ \\
\hline População & $\begin{array}{c}\mathrm{X}_{2} \\
\text { Variável } \\
\text { independente }\end{array}$ & $\begin{array}{l}\text { Número total de residentes, } \\
\text { independentemente do seu estatuto } \\
\text { legal e/ ou cidadania. }\end{array}$ & $\begin{array}{c}\text { World Bank } \\
\text { (www.worldbank.org) }\end{array}$ \\
\hline Tempo & $\begin{array}{c}\mathrm{X}_{3} \\
\text { Variável } \\
\text { independente }\end{array}$ & $\begin{array}{l}\text { Representa a unidade temporal que } \\
\text { reportam os dados em análise, o ano } \\
\text { civil (indexação do ano civil às várias } \\
\text { variáveis em estudo). }\end{array}$ & Elaboração Própria \\
\hline $\begin{array}{l}\text { Preço da } \\
\text { Passagem } \\
\text { Aérea }\end{array}$ & $\begin{array}{c}\mathrm{X}_{4} \\
\text { Variável } \\
\text { independente }\end{array}$ & $\begin{array}{l}\text { Representa a média ponderada dos } \\
\text { preços das passagens aéreas } \\
\text { (considerando o volume de passageiros } \\
\text { e a respectiva tarifa entre origem e } \\
\text { destino O-D) sem a contabilização das } \\
\text { taxas aeroportuárias e expressa em } \\
\text { dólares americanos }(\$) \text {. }\end{array}$ & $\begin{array}{l}\text { Sabre Airport Data } \\
\text { Intelligence } \\
\text { (www.airdi.net) }\end{array}$ \\
\hline $\begin{array}{l}\text { Factor de } \\
\text { Negócio }\end{array}$ & $\begin{array}{c}\mathrm{X}_{5} \\
\text { Variável } \\
\text { independente }\end{array}$ & $\begin{array}{l}\text { Representa uma variável dummy, } \\
\text { identificando com o algarismo (1) o } \\
\text { destino que contiver um factor de } \\
\text { atracção de negócio (classificação } \\
\text { baseada em informações da Embaixada } \\
\text { da República Popular da China, em } \\
\text { Portugal), caso contrário identificará o } \\
\text { mesmo com o algarismo (0). }\end{array}$ & Elaboração Própria \\
\hline $\begin{array}{c}\text { Factor de } \\
\text { Turismo }\end{array}$ & $\begin{array}{c}\mathrm{X}_{6} \\
\text { Variável } \\
\text { independente }\end{array}$ & $\begin{array}{l}\text { Representa uma variável dummy, } \\
\text { identificando com o algarismo (1) o } \\
\text { destino que contiver um factor de } \\
\text { atracção de turismo (classificação } \\
\text { baseada em informações da Embaixada } \\
\text { da República Popular da China, em } \\
\text { Portugal), caso contrário identificará o } \\
\text { mesmo com o algarismo (0). }\end{array}$ & Elaboração Própria \\
\hline Passageiros & $\begin{array}{c}\text { Y } \\
\text { Variável } \\
\text { dependente }\end{array}$ & $\begin{array}{l}\text { Número de passageiros transportados } \\
\text { anualmente em cada uma das rotas } \\
\text { estudadas. }\end{array}$ & $\begin{array}{l}\text { Sabre Airport Data } \\
\text { Intelligence } \\
\text { (www.airdi.net) }\end{array}$ \\
\hline
\end{tabular}


As variáveis dummy, nomeadamente o Factor de Negócio e o Factor de Turismo, elaborados pelo autor deste trabalho de investigação pretendem caracterizar como cada um dos destinos estudados no modelo valoriza o aumento de passageiros, verificando-se, dessa forma, se um determinado local possui um interesse negocial e/ ou turístico para os diversos passageiros que usufruem de cada uma das rotas em análise.

Depois de expressas as relações entre as variáveis independentes e a corresponde variável independente, assim como a apresentação e caracterização de todas as variáveis utilizadas, é necessário definir o modelo de regressão linear múltipla através das seguintes equações. A Equação 3 é respeitante ao primeiro modelo enquanto a Equação 4 é respeitante ao segundo modelo em estudo.

$$
\text { Modelo } 1 \rightarrow \ln \mathrm{Y}_{\mathrm{ijt}}=\mathrm{c}+\beta_{1} \ln \mathrm{X}_{1 \mathrm{ij}}+\beta_{2} \ln \mathrm{X}_{2 \mathrm{ij}}+\beta_{3} \ln \mathrm{X}_{3 \mathrm{ij}}+\varepsilon_{\mathrm{ij}}
$$

$$
\mathrm{t}=\text { período de tempo em análise }=[1 ; 8]
$$

$\mathrm{ij}=$ foram realizadas 8 observações para cada uma das 25 rotas $=[1 ; 25]$

$$
\varepsilon_{\mathrm{ijt}}=\alpha_{\mathrm{ij}}+\mathrm{u}_{\mathrm{ij} \mathrm{t}}
$$

A Equação 4 é bastante semelhante à Equação 3, diferindo apenas na quantidade de variáveis independentes seleccionadas ao longo do trabalho de investigação.

$$
\begin{aligned}
& \text { Modelo } 2 \rightarrow \ln \mathrm{Y}_{\mathrm{ijt}}=\mathrm{c}+\beta_{1} \ln \mathrm{X}_{1 \mathrm{ij}}+\beta_{2} \ln \mathrm{X}_{2 \mathrm{ij}}+\beta_{3} \ln \mathrm{X}_{3 \mathrm{ij}}+\beta_{4} \ln \mathrm{X}_{4 \mathrm{ij}}+\beta_{5} \ln \mathrm{X}_{5 \mathrm{ij} \mathrm{t}} \\
& \qquad+\beta_{6} \ln \mathrm{X}_{6 \mathrm{ij}}+\varepsilon_{\mathrm{ij} \mathrm{t}} \\
& \mathrm{t}=\text { período de tempo em análise }=[1 ; 8] \\
& \mathrm{ij}=\text { foram realizadas } 8 \text { observações para cada uma das } 25 \text { rotas }=[1 ; 25] \\
& \qquad \varepsilon_{\mathrm{ij}}=\alpha_{\mathrm{ij}}+\mathrm{u}_{\mathrm{ij}} \mathrm{t}
\end{aligned}
$$

Através da Equação 3 e da Equação 4 é necessário efectuar as seguintes ressalvas para uma melhor compreensão de todos os membros que a constituem, nomeadamente: 
- $\quad \alpha_{\mathrm{ij}}$ representa factores específicos e também aqueles de difícil observação em cada uma das rotas estudadas;

- $\mathrm{u}_{\mathrm{ij} t}$ representa os erros que podem, de alguma forma, influenciar de forma directa e/ ou indirecta os resultados obtidos através do modelo definido anteriormente;

- A soma dos dois parâmetros apresentados anteriormente representam a variabilidade a que a variável dependente está sujeita e que não pode ser explicada através das diversas variáveis independentes escolhidas para a definição do modelo. Como tal, quanto menor for este valor melhor será o modelo, visto que consegue representar de forma fidedigna o comportamento do fluxo de passageiros.

\section{Análise de dados e discussão}

Recorrendo às Equações 3 e 4 pretende-se modelar um conjunto de dados apresentados nas Tabelas 1 e 2, e encontrar o modelo que melhor traduz essa modelação, através dos métodos de estimação por pooled Ordinary Least Squares (OLS), por efeitos fixos e por efeitos aleatórios (Schmidheiny, 2012).

Esta secção é de grande importância, visto que representa o culminar de todo o processo de investigação realizado, contendo a análise de cada um dos dois modelos de regressão linear múltipla apresentados anteriormente, contendo a apresentação dos resultados obtidos e a consequente comparação dos mesmos, mediante os diversos métodos de estimação:

\subsection{Resultados do primeiro modelo}

Antes da apresentação das tabelas correspondentes aos resultados obtidos através da utilização do primeiro modelo para o estudo da variação do fluxo de passageiros, é necessário definir os seus modelos matemáticos recorrendo a um modelo de regressão linear múltipla contendo dados de painel. A definição do Modelo 1 encontra-se representada através da Equação 5 e dos resultados obtidos através do mesmo, tendo em conta os métodos de estimação referidos anteriormente, encontram-se expressos na Tabela 3.

$\ln$ Passageiros $_{i j} t=c+\beta_{1} \ln \operatorname{PIB}_{i j} t+\beta_{2} \ln$ População $_{i j t}+\beta_{3} \ln \mathrm{Tempo}_{i j t}+\alpha_{i j}+u_{i j} t$ 


\section{Tabela 3 - Resultados obtidos através do Modelo 1}

\begin{tabular}{|c|c|c|c|}
\hline & Pooled OLS & Efeitos Aleatórios & Efeitos Fixos \\
\hline $\begin{array}{c}\text { Variáveis } \\
\text { Independentes }\end{array}$ & \multicolumn{3}{|c|}{$\begin{array}{l}\text { Coeficiente, erro-padrão, estatísticas de teste, } \mathbf{R}^{2}, \text { níveis de } \\
\text { significância estatística }(*=1 \%, * *=5 \%, * * *=10 \%)\end{array}$} \\
\hline \multirow{2}{*}{$\begin{array}{c}\text { Constante } \\
\text { (Erro-Padrão com } \\
\text { Heterocedasticidade } \\
\text { corrigida) }\end{array}$} & 1891,859 & 176,4536 & 49,9977 \\
\hline & 464,5447 & 251,9574 & 233,4162 \\
\hline \multirow{2}{*}{$\begin{array}{c}\text { PIB } \\
\text { (Erro-Padrão com } \\
\text { Heterocedasticidade } \\
\text { corrigida) }\end{array}$} & $0,1713532 * / * * / * *$ & $0,0429625 * * / * * *$ & $0,0364289 * * / * * *$ \\
\hline & 0,0332483 & 0,0185668 & 0,0171909 \\
\hline \multirow{2}{*}{$\begin{array}{c}\text { População } \\
\text { (Erro-Padrão com } \\
\text { Heterocedasticidade } \\
\text { corrigida) }\end{array}$} & $0,1951739 * / * * / * *$ & $0,0702127 * * / * * *$ & $2,175919 * / * * / * * *$ \\
\hline & 0,033737 & 0,0245342 & 0,347676 \\
\hline \multirow{2}{*}{$\begin{array}{c}\text { Tempo } \\
\text { (Erro-Padrão com } \\
\text { Heterocedasticidade } \\
\text { corrigida) }\end{array}$} & $(0,9721759) * / * * / * *$ & $(0,0903849)$ & $0,3225383 * * / * * *$ \\
\hline & 0,2380408 & 0,129344 & 0,1351754 \\
\hline $\mathrm{R}^{2}$ & 0,2190 & 0,1578 & 0,0798 \\
\hline \multirow[t]{2}{*}{ Estimação do rho } & --- & 0,91513688 & 0,99989453 \\
\hline & $\begin{array}{c}\mathrm{F}(3,196)=18,32 \\
\text { pvalue }(\text { Prob }>F=0,00)\end{array}$ & $\begin{array}{c}\text { Wald } \mathrm{Chi}^{2}(3)=90,54 \\
\text { pvalue }\left(\text { Prob }>\mathrm{Chi}^{2}=0,00\right)\end{array}$ & $\begin{array}{c}\mathrm{F}(3,172)=47,05 \\
\text { pvalue }(\text { Prob }>\mathrm{F}=0,00)\end{array}$ \\
\hline
\end{tabular}

De acordo com a Tabela 3 é possível concluir que o Modelo 1 no método de estimação por pooled OLS produz os melhores resultados, tendo em conta os seguintes dados e ressalvas importantes para uma adequada interpretação dos resultados obtidos:

- Com a variação percentual de cada uma das variáveis explicativas, mantendo todas as outras constantes, a variável dependente, isto é, o número de passageiros, possui a seguinte variação: aumento de 17,1\% devido ao aumento da variável correspondente ao PIB; acréscimo de 19,5\% 
devido ao aumento da variável correspondente à População; e, decréscimo de 97,2\% devido ao aumento da variável correspondente ao Tempo;

- É possível então afirmar que apenas a variável correspondente ao PIB e População possuem a capacidade de promover um efeito positivo para a variação do número de passageiros, sendo que a restante tem uma relação contrária;

- É importante analisar e interpretar se os coeficientes da regressão são, na realidade, significativos ou não para o modelo em estudo, sabendo que quanto menor o seu nível de significância, maior será a sua probabilidade de impacto na variação da variável dependente e também confiança nos resultados obtidos. Relativamente aos três níveis de significância analisados, $1 \%$, 5\% e 10\% (Tabela 3), podemos concluir que o método de estivação por pooled OLS apresenta melhores resultados comparando com os métodos de estimação por efeitos fixos e por efeitos aleatórios.

- A heterocedasticidade é um fenómeno observado em cada modelo quando as variâncias obtidas para todas as variáveis, seja dependente e/ ou independentes, são distintas entre si, verificandose dessa forma uma dispersão dos dados em torno da recta de regressão. Para cada método de estimação foi implementada uma medida correctiva com o objectivo de ser obtido o erro-padrão com heterocedasticidade corrigida de acordo com o teste White para cada uma das variáveis em análise nos modelos;

- O parâmetro representado por $\mathrm{R}^{2}$ é denominado por coeficiente de determinação e tem como objectivo demonstrar o valor percentual da variação da variável dependente que pode ser explicada a partir do valor das variáveis independentes e, como tal, varia entre 0 e 1 . O restante valor dessa percentagem para a totalidade corresponde, em contrapartida, ao valor percentual explicado por outros parâmetros que não foram considerados no modelo. Através do valor obtido é possível afirmar que as variáveis independentes que constituem este modelo têm a capacidade de explicar $21,90 \%$ da variação do número de passageiros;

- Através do teste da estatística F-Snedcor é possível verificar a existência de uma relação entre a variável dependente e as diversas variáveis independentes, verificando-se que quando unidas, as variáveis independentes têm um peso de 18,32 a um nível de significância de $1 \%$.

\subsection{Resultados do segundo modelo}

Antes da apresentação das tabelas correspondentes aos resultados obtidos através da utilização do primeiro modelo para o estudo da variação do fluxo de passageiros, é necessário relembrar os seus modelos matemáticos recorrendo a um modelo de regressão linear múltipla contendo dados de painel. 
A definição do Modelo 2 encontra-se representada através da Equação 7 e dos resultados obtidos através do mesmo, tendo em conta os métodos de estimação referidos anteriormente, encontram-se expressos na Tabela 4.

$\ln$ Passageiros $_{\mathrm{ij}} \mathrm{t}=\mathrm{c}+\beta_{1} \ln \mathrm{PIB}_{\mathrm{ij}}+\beta_{2} \ln$ População $_{\mathrm{ij}} \mathrm{t}+\beta_{3} \ln$ Preço da Passagem

$$
\text { Aérea }_{\mathrm{ij} t}+\beta_{4} \ln \text { Factor de Negócio }_{\mathrm{ij}} \mathrm{t}+\beta_{5} \ln \text { Factor de Turismo }_{\mathrm{ij}} \mathrm{t}+\beta_{6} \ln \mathrm{Tempo}_{\mathrm{ij}} \mathrm{t}
$$

$$
+\alpha_{i j}+u_{i j t}
$$

De acordo com a Tabela 4 é possível concluir que o Modelo 2 no método de estimação por pooled OLS produz os melhores resultados, tendo em conta os seguintes dados e ressalvas importantes para uma adequada interpretação dos resultados obtidos:

- Com a variação percentual de cada uma das variáveis explicativas, mantendo todas as outras constantes, a variável dependente, isto é, o número de passageiros, possui a seguinte variação: aumento de 15,4\% devido ao aumento da variável correspondente ao PIB; acréscimo de 16,4\% devido ao aumento da variável correspondente à População; decréscimo de 53,9\% devido ao aumento da variável correspondente ao Preço da Passagem Aérea; aumento de 243,9\% devido ao aumento da variável correspondente ao Factor de Negócio; aumento de 163,3\% devido ao aumento da variável correspondente ao Factor de Turismo; e, decréscimo de 84,1\% devido ao aumento da variável correspondente ao Tempo.

- É possível então afirmar que as variáveis correspondentes ao PIB, População, Factor de Negócio e Factor de Turismo possuem a capacidade de promover um efeito positivo para a variação do número de passageiros, resultados em linha com as principais correntes da literatura face ao tema, abordadas na primeira secção do presente artigo, sendo que as restantes têm uma relação contrária.

- É importante analisar e interpretar se os coeficientes da regressão são, na realidade, significativos ou não para o modelo em estudo, sabendo que quanto menor o seu nível de significância, maior será a sua probabilidade de impacto na variação da variável dependente e também confiança nos resultados obtidos. Para o efeito, analisou-se os dados para os três níveis de significância, $1 \%, 5 \%$ e $10 \%$ (Tabela 4).

- Através do valor obtido para o coeficiente de determinação é possível afirmar que as variáveis independentes que constituem este modelo têm a capacidade de explicar $28,10 \%$ da variação do número de passageiros; 
Tabela 4 - Resultados obtidos através do Modelo 2

\begin{tabular}{|c|c|c|c|}
\hline & Pooled OLS & Efeitos Aleatórios & Efeitos Fixos \\
\hline $\begin{array}{c}\text { Variáveis } \\
\text { Independentes }\end{array}$ & \multicolumn{3}{|c|}{$\begin{array}{l}\text { Coeficiente, erro-padrão, estatísticas de teste, } \mathbf{R}^{2}, \text { níveis de significância } \\
\text { estatística }(*=1 \%, * *=5 \%, * * *=10 \%)\end{array}$} \\
\hline \multirow{2}{*}{$\begin{array}{c}\text { Constante } \\
\text { (Erro-Padrão com } \\
\text { Heterocedasticidade } \\
\text { corrigida) }\end{array}$} & 1632,484 & 160,1387 & $(427,6384)$ \\
\hline & 455,429 & 252,6535 & 41,8624 \\
\hline \multirow{2}{*}{$\begin{array}{c}\text { PIB } \\
\text { (Erro-Padrão com } \\
\text { Heterocedasticidade } \\
\text { corrigida) }\end{array}$} & $0,1543249 * / * * / * * *$ & $0,0414561 * * / * * *$ & $0,112275 * * *$ \\
\hline & 0,0325403 & 0,0186468 & 0,0136945 \\
\hline \multirow{2}{*}{$\begin{array}{c}\text { População } \\
\text { (Erro-Padrão com } \\
\text { Heterocedasticidade } \\
\text { corrigida) }\end{array}$} & $0,1638533 * / * * / * * *$ & $0,0552316 * * *$ & $2,175375 * / * * / * * *$ \\
\hline & 0,0339857 & 0,0288715 & 0,3506875 \\
\hline \multirow{2}{*}{$\begin{array}{l}\text { Preço da Passagem } \\
\text { Aérea } \\
\text { (Erro-Padrão com } \\
\text { Heterocedasticidade } \\
\text { corrigida) }\end{array}$} & $(0,5387464)$ & 0,1033738 & 0,176742 \\
\hline & 0,3680573 & 0,1468285 & 0,1354267 \\
\hline \multirow{2}{*}{$\begin{array}{c}\text { Factor de Negócio } \\
\text { (Erro-Padrão com } \\
\text { Heterocedasticidade } \\
\text { corrigida) }\end{array}$} & $2,439658 * / * * / * * *$ & 2,11874 & $1,98403 * * *$ \\
\hline & 0,6158292 & 1,894282 & 1,746830 \\
\hline \multirow{2}{*}{$\begin{array}{l}\text { Factor de Turismo } \\
\text { (Erro-Padrão com } \\
\text { Heterocedasticidade } \\
\text { corrigida) }\end{array}$} & $1,632859 * * / * * *$ & 1,373683 & 1,280473 \\
\hline & 0,5745739 & 1,782271 & 1,673042 \\
\hline \multirow{2}{*}{$\begin{array}{c}\text { Tempo } \\
\text { (Erro-Padrão com } \\
\text { Heterocedasticidade } \\
\text { corrigida) }\end{array}$} & $(0,8411784) * / * * / * * *$ & $(0,0855822)$ & $0,5733653 * / * * / * * *$ \\
\hline & 0,2333016 & 0,1296228 & 0,0643459 \\
\hline $\mathrm{R}^{2}$ & 0,2810 & 0,2196 & 0,0793 \\
\hline \multirow[t]{2}{*}{ Estimação do rho } & --- & 0,91760287 & 0,99989644 \\
\hline & $\begin{array}{c}\mathrm{F}(6,193)=12,57 \\
\text { pvalue }(\text { Prob }>F=0,00)\end{array}$ & $\begin{array}{c}\text { Wald } \mathrm{Chi}^{2}(6)=92,07 \\
\text { pvalue }\left(\text { Prob }>\mathrm{Chi}^{2}=0,00\right)\end{array}$ & $\begin{array}{c}\mathrm{F}(3,172)=45,40 \\
\text { pvalue }(\text { Prob }>F=0,00)\end{array}$ \\
\hline
\end{tabular}


Através do teste da estatística F-Snedcor é possível verificar a existência de uma relação entre a variável dependente e as diversas variáveis independentes, verificando-se que quando unidas, as variáveis independentes têm um peso de 12,57 a um nível de significância de $1 \%$.

\subsection{Comparação entre os métodos de estimação dos dois modelos}

Apesar de todas as diferenças e, em alguns casos, semelhanças entre os dois modelos analisados neste trabalho de investigação, tendo em conta os respectivos métodos de estimação, é importante referir, primeiramente, que a escolha do melhor modelo de estimação deve contemplar os objectivos do estudo em que se inserem os modelos em comparação, o tipo de variáveis que os constituem e toda a envolvente em que se inserem.

Por outro lado, e de forma sucinta, é possível afirmar que o segundo modelo é aquele que promove uma melhor e adequada explicação quanto à variação do número de passageiros. Apesar desta conclusão é importante referir que o primeiro modelo, apesar da sua simplicidade, das suas características próprias e das suas limitações quando comparado com o segundo modelo, promove resultados positivos como era esperado antes da realização deste estudo, visto que as diferenças não se encontram presentes na tendência da influência (positiva ou negativa) mas sim no peso dessa mesma influência, isto é, os valores dos coeficientes da regressão.

A conclusão expressa anteriormente baseou-se numa análise detalhada e consistente ao longo de todos os parâmetros obtidos através deste mesmo estudo, visto que a escolha do modelo e consequente método de estimação não se deve basear única e exclusivamente em um dos parâmetros, como por exemplo, o facto de determinado método de estimação do modelo de regressão possuir um elevado carácter significativo não implica, necessariamente, que o mesmo represente o método de estimação mais adequado para o problema em análise. Este conselho é de extrema importância e utilidade para que não sejam obtidas conclusões precipitadas e, muitas vezes, incorrectas.

De entre as diversas variáveis independentes que constituem o modelo é importante salientar o efeito benéfico dos dois factores elaborados pelo autor, nomeadamente o Factor de Negócio e o Factor de Turismo (variáveis dummy comportamentais do motivo da viagem/atracção do destino para o mercado Chinês), assim como do PIB, da População, promovendo as mesmas, um contributo bastante positivo para o crescimento do número de passageiros, independentemente do método de estimação em análise.

Por outro lado, através da análise do parâmetro $\mathrm{R}^{2}$ verifica-se que aproximadamente $29 \%$ da variação do número de passageiros é explicada através das sete variáveis independentes. Uma conclusão semelhante é obtida através da análise do parâmetro R, que corresponde ao coeficiente de Pearson e tem 
o valor de aproximadamente 0,53 , sendo que através do mesmo se verifica uma intensidade significativa da relação linear existente entre as diversas variáveis e, como tal, quando ocorre uma variação positiva das mesmas, tendencialmente o número de passageiros também irá sofrer um crescimento.

Através da bibliografia consultada é importante referir as conclusões obtidas por estudos realizados no âmbito em que se insere este trabalho de investigação, apesar da inexistência de uma vasta variedade de bibliografia mundial, o que indica que a metodologia utilizada não se encontra aplicada nos estudos como seria desejável, mesmo depois de comprovada a sua simplicidade e benefícios promovidos.

No caso de Costa et al. (2008), os autores debruçaram os seus esforços na análise da implicação do tipo de infra-estrutura aeroportuária para a influência do fluxo de passageiros, obtendo-se dessa forma modelos de regressão adequados a vários tipos de infra-estruturas aeroportuárias, nomeadamente as que se encontram localizadas em cidades do litoral do Brasil, assim como para cidades potencialmente imprescindíveis para a realização de negócios (Costa et al., 2008).

Por outro lado, e mais recentemente, Condé (2011) desenvolveu um estudo que permitiu analisar a viabilidade da potencialidade de crescimento dos aeroportos do Rio de Janeiro, construindo para isso modelos de regressão linear, efectuando uma previsão quanto ao fluxo de passageiros (Condé, 2011).

Apesar de se ter concluído no presente trabalho de investigação a influência do PIB para a variação positiva do fluxo de passageiros, Rocha (2011) conclui que esta relação não era tão intensa quando se tinham em conta infra-estruturas aeroportuárias localizadas em regiões com menor grau de desenvolvimento socioeconómico, particularidade que não se encontra patente no caso das infraestruturas aeroportuárias analisadas para a criação dos dois modelos (Rocha, 2011). De acordo com este mesmo estudo é possível concluir a importância da realização de investimentos apropriados nestas infraestruturas de modo a impulsionar o seu crescimento e potencialidade para novas rotas.

\section{Nível de circuito e a potencialidade da exploração do mercado Chinês}

A partir dos resultados expostos anteriormente para cada um dos modelos e tendo em conta que se trata de rotas originárias de três dos principais aeroportos brasileiros com destino a aeroportos asiáticos, é possível confirmar a elevada influência da actividade do sector do transporte aéreo no que diz respeito ao fluxo elevado de passageiros. Essa influência poderia facilmente beneficiar o número de passageiros em movimento no aeroporto internacional de Lisboa tendo em conta, por exemplo, a existência de rotas originárias através do mesmo e com destino a cidades chinesas, tal como se pode ver através da seguinte tabela (Tabela 5). 
Tabela 5 - Número de passageiros transportados entre Lisboa e China no ano de 2010 ${ }^{1}$

\begin{tabular}{|c|c|c|}
\hline $\begin{array}{c}\text { Aeroporto de Partida } \\
\text { (Código IATA) }\end{array}$ & $\begin{array}{c}\text { Aeroporto de Chegada } \\
\text { (Código IATA) }\end{array}$ & $\begin{array}{c}\text { Número de Passageiros } \\
\text { Transportados }\end{array}$ \\
\hline Lisboa (LIS) & Shangai (PVG) & 21899 \\
\hline Lisboa (LIS) & Hong Kong (HKG) & 20575 \\
\hline Lisboa (LIS) & Pequim (PEK) & 11582 \\
\hline
\end{tabular}

A Tabela 5 pretende, de forma simplificada, sugerir as oportunidades de transferência de tráfego, nomeadamente através da exploração de uma rota entre Lisboa e Hong-Kong, seja através de voos directos ou então ao abrigo de voos realizados em code-share, fazendo com que, por exemplo, duas companhias de transporte aéreo unam esforços para a realização deste tipo de voos, tal como já se verifica na actualidade entre a TAP Portugal e a Air China.

Por outro lado, através da análise dos dados que constituem os dois modelos em estudo, e consequente análise na Tabela 5, é possível elaborar o estudo de um parâmetro ainda pouco referenciado ao longo da literatura desta temática, isto é, o nível de circuito, sendo que este parâmetro se obtém através do cálculo expresso na seguinte equação (Equação 8).

Nível de Circuito $=[($ Distância da Origem-Hub+Distância Hub-

\section{Destino)/Distância Origem-Destino]}

Apesar deste parâmetro não se encontrar aplicado a um vasto conjunto de estudos no âmbito do sector aeronáutico, Borenstein desenvolveu uma análise de forma a determinar a importância da rota e infraestruturas aeroportuárias para a determinação da influência e poder que cada companhia aérea exerce no mercado em que se insere (Borenstein, 1989). Através do estudo realizado foi possível verificar que a escolha das rotas e aeroportos definidos para criação de hubs tinham uma grande influência para a variação dos custos suportados pelas companhias aéreas (Borenstein, 1989). Concluiu-se, dessa forma, que o preço para a operação em determinada rota tem um crescimento directamente relacionado com o volume de tráfego que opera na mesma mas que, em contrapartida, este fenómeno não poderia ser claramente evidenciado e explicado através das metodologias utilizadas até então, verificando-se a importância do parâmetro que diz respeito ao nível de circuito (Borenstein, 1989).

\footnotetext{
${ }^{1}$ Fonte: Sabre Airport Data Intelligence (www.airdi.net).
} 
Tabela 6 - Exemplificação de 33 rotas e o correspondente nível de circuito

\begin{tabular}{|c|c|c|c|c|c|c|}
\hline $\begin{array}{c}\text { Rota } \\
\text { (Códigos IATA) }\end{array}$ & $\begin{array}{l}\text { Número } \\
\text { da Rota }\end{array}$ & $\begin{array}{l}\text { Distância da } \\
\text { Origem ao Hub } \\
(\mathrm{Km})\end{array}$ & $\begin{array}{c}\text { Distância do } \\
\text { Hub ao Destino } \\
(\mathrm{Km})\end{array}$ & $\begin{array}{c}\text { Distância } \\
\text { da Origem } \\
\text { ao Destino } \\
(\mathrm{Km})\end{array}$ & $\begin{array}{l}\text { Nível de } \\
\text { Circuito }\end{array}$ & $\begin{array}{l}\text { Número de } \\
\text { Passageiros }\end{array}$ \\
\hline GRU-DOH-HKG & 1 & 11881 & 6328 & 18064 & 1,008 & 701 \\
\hline GIG-DOH-HKG & 2 & 11533 & 6328 & 17718 & 1,008 & 23 \\
\hline POA-DOH-HKG & 3 & 12549 & 6328 & 18341 & 1,029 & 24 \\
\hline GRU-DXB-HKG & 4 & 12241 & 5957 & 18064 & 1,007 & 1513 \\
\hline GIG-DXB-HKG & 5 & 11892 & 5957 & 17718 & 1,007 & 36 \\
\hline POA-DXB-HKG & 6 & 12896 & 5957 & 18341 & 1,028 & 112 \\
\hline GRU-JFK-HKG & 7 & 7672 & 12977 & 18064 & 1,143 & 240 \\
\hline GIG-JFK-HKG & 8 & 7738 & 12977 & 17718 & 1,169 & 333 \\
\hline POA-JFK-HKG & 9 & 8206 & 12977 & 18341 & 1,155 & 4 \\
\hline GRU-MIA-HKG & 10 & 6578 & 14454 & 18064 & 1,164 & 19 \\
\hline GIG-MIA-HKG & 11 & 6724 & 14454 & 17718 & 1,195 & 6 \\
\hline POA-MIA-HKG & 12 & 6949 & 14454 & 18341 & 1,167 & 13 \\
\hline GRU-LAX-HKG & 13 & 9919 & 11654 & 18064 & 1,194 & 170 \\
\hline GIG-LAX-HKG & 14 & 10148 & 11654 & 17718 & 1,231 & 1 \\
\hline POA-LAX-HKG & 15 & 10027 & 11654 & 18341 & 1,182 & 1 \\
\hline GRU-LIS-HKG & 16 & 7955 & 11031 & 18064 & 1,051 & 6 \\
\hline GIG-LIS-HKG & 17 & 7722 & 11031 & 17718 & 1,058 & 17 \\
\hline POA-LIS-HKG & 18 & 8816 & 11031 & 18341 & 1,082 & 1 \\
\hline GRU-FRA-HKG & 19 & 9821 & 9178 & 18064 & 1,052 & 1175 \\
\hline GIG-FRA-HKG & 20 & 9578 & 9178 & 17718 & 1,059 & 65 \\
\hline POA-FRA-HKG & 21 & 10681 & 9178 & 18341 & 1,083 & 197 \\
\hline GRU-MAD-HKG & 22 & 8399 & 10536 & 18064 & 1,048 & 91 \\
\hline GIG-MAD-HKG & 23 & 8154 & 10536 & 17718 & 1,055 & 9 \\
\hline POA-MAD-HKG & 24 & 9258 & 10536 & 18341 & 1,079 & 7 \\
\hline GRU-CDG-HKG & 25 & 9427 & 9615 & 18064 & 1,054 & 1706 \\
\hline GIG-CDG-HKG & 26 & 9194 & 9615 & 17718 & 1,062 & 927 \\
\hline POA-CDG-HKG & 27 & 10288 & 9615 & 18341 & 1,085 & 98 \\
\hline GRU-LHR-HKG & 28 & 9482 & 9654 & 18064 & 1,059 & 330 \\
\hline GIG-LHR-HKG & 29 & 9263 & 9654 & 17718 & 1,068 & 189 \\
\hline POA-LHR-HKG & 30 & 10341 & 9654 & 18341 & 1,090 & 20 \\
\hline GRU-AMS-HKG & 31 & 9799 & 9297 & 18064 & 1,057 & 414 \\
\hline GIG-AMS-HKG & 32 & 9570 & 9297 & 17718 & 1,065 & 7 \\
\hline POA-AMS-HKG & 33 & 10658 & 9297 & 18341 & 1,088 & 34 \\
\hline & & & & & TOTAL & 8489 \\
\hline
\end{tabular}

\footnotetext{
${ }^{2}$ Fonte: Sabre Airport Data Intelligence (www.airdi.net) e Airport Codes Database and Airports Distance Calculator (www.airportcitycodes.com/).
} 
Com um intuito meramente exemplificativo, a tabela seguinte (Tabela 6) contém a descrição de 33 rotas dando origem à Figura 1 que contém a apresentação da importância do nível de circuito nas correspondentes rotas. As rotas referidas anteriormente têm como intuito fazer uma ligação entre três das principais infra-estruturas aeroportuárias brasileiras e a cidade de Hong Kong, testando para isso 11 ligações distintas, tendo como objectivo verificar a potencialidade de uma rota entre estes dois mercados, via Lisboa, e que não existe na actualidade.

A partir da tabela anterior é possível verificar distintos valores para o parâmetro denominado por nível de circuito e, como tal, é necessário efectuar algumas ressalvas quanto ao seu significado e importância, para além da sua metodologia de cálculo que já foi anteriormente expressa. Acima de tudo, o mesmo permite efectuar uma avaliação quanto à qualidade geográfica da infra-estrutura aeroportuária que serve de hub para cada uma das rotas. Caso o valor seja reduzido e, principalmente próximo do valor unitário, é possível afirmar que o desvio efectuado por um determinado passageiro entre um par de cidades é minimizado e que a respectiva companhia aérea em que viaja tem a capacidade de promover uma vantagem relativamente ao tempo necessário para efectuar a deslocação. Mas é importante referir que este parâmetro sofre uma grande influência dos tempos necessários para a realização da transferência de passageiros e/ou bagagens nos respectivos hubs (Grimme, 2011).

A potencialidade referida anteriormente é facilmente perceptível através da Tabela 6 e tendo em conta que não só o tempo de transferência como também a frequência de voos constituem um factor bastante significativo para a escolha das companhias por parte dos passageiros, sabendo-se dessa forma que as companhias da Star Alliance possuem uma maior frequência de voos para a região asiática e, dessa forma, aumentando a flexibilidade para determinados tipos de passageiros, nomeadamente aqueles que efectuam as suas deslocações em trabalho.

A figura seguinte (Figura 1) tem como intenção exemplificar através de uma evolução gráfica do nível de circuito promovido por determinadas rotas e, através do mesmo, é possível verificar que as rotas identificadas pelos algarismos 1, 2, 4 e 5 são as mais benéficas para o parâmetro em análise, correspondendo, respectivamente, ao hub de Doha, no Qatar, e ao hub do Dubai, nos Emirados Árabes Unidos, sendo importante referir a proximidade geográfica que os mesmos têm ao aeroporto internacional de Lisboa.

Apesar das rotas exemplificativas que passam pelo aeroporto internacional de Lisboa na Figura 1 não possuírem valores tão favoráveis, é importante verificar a proximidade que as mesmas possuem do valor unitário e das potencialidades existentes neste aeroporto que não se encontram presentes nos aeroportos citados anteriormente, nomeadamente, o montante das tarifas e até mesmo os tempos de rotação exercidos nos mesmos. Através da análise do nível de circuito aplicado para explorar a potencialidade 
do mercado Chinês, em termos de análise de parâmetro estratégico da Star Alliance, aliança de companhias aéreas em que a companhia aérea portuguesa TAP Portugal possue uma posição estratégica no mercado do Brasil e tendo o seu principal hub no aeroporto internacional de Lisboa, perante os resultados obtidos, considera-se uma sugestão pertinente e viável a exploração das rotas acima indicadas para o mercado Chinês, via Lisboa.

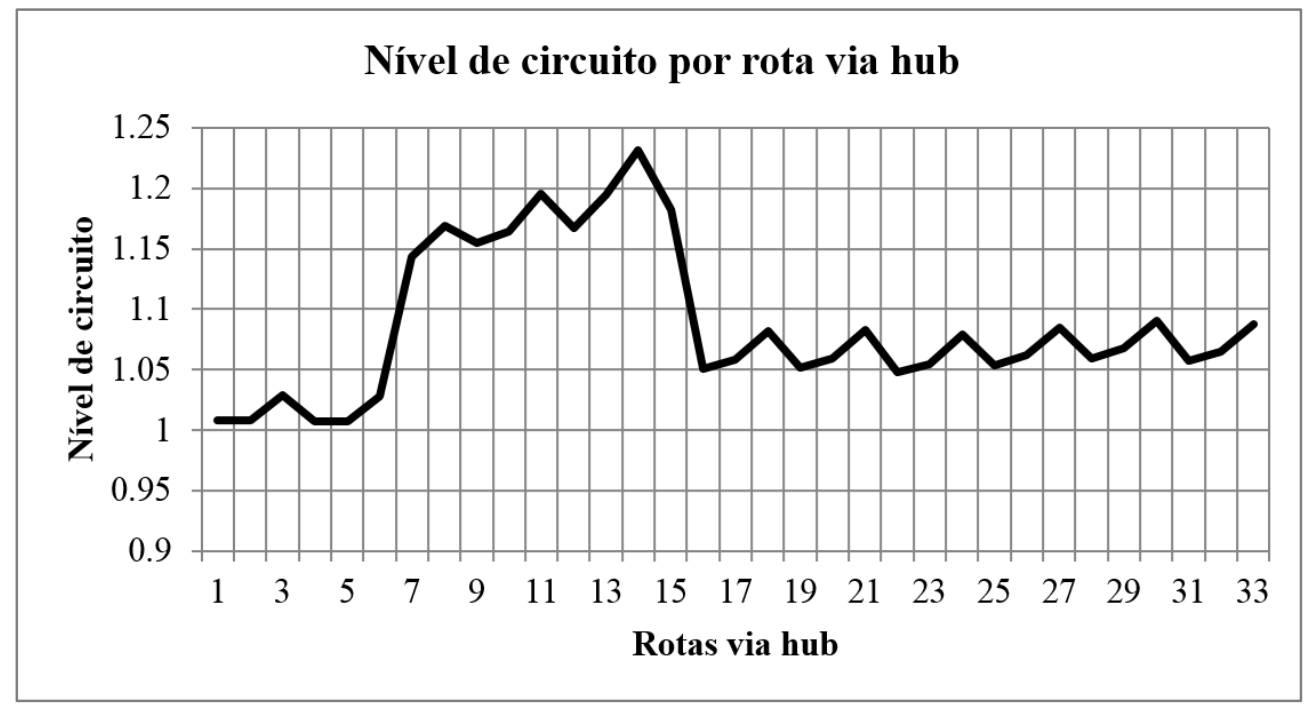

Figura 1 - Nível de circuito por rota via hub

\section{Conclusão}

Tendo como ponto de partida a necessidade de analisar e confirmar a potencialidade do mercado da aviação chinesa para o desenvolvimento e crescimento do aeroporto internacional de Lisboa, procedeuse a uma análise desta problemática recorrendo a dois modelos de regressão linear múltipla, também designados por modelos gravitacionais com dados de painel, sendo cada um deles caracterizado de forma particular e objectiva.

Através da construção dos dois modelos, pretendeu-se traduzir a variação do fluxo de passageiros como uma função dependente de vários conjuntos pré-estabelecidos de variáveis que, de forma mais ou menos directa, pretendiam explicar como a sua variação afectava o número de passageiros transportados através do sector do transporte aéreo nas diversas rotas seleccionadas.

Os resultados obtidos evidenciaram claramente o potencial desse mercado para o aeroporto internacional de Lisboa, nomeadamente através do segundo modelo (modelo 2) de regressão linear múltipla, sendo aquele que, para além de uma maior quantidade de variáveis que compõem o modelo, possuem ainda as 
variáveis dummy como pretexto para explicação da influência do interesse negocial e turístico de cada uma das rotas para os passageiros que usufruem das mesmas.

Por outro lado, através da metodologia em análise, foi possível elaborar um conjunto de referências que justifiquem o desenvolvimento de rotas que utilizem como aeroporto de origem e/ ou aeroporto de hub o aeroporto internacional de Lisboa, tendo em conta o estudo secundário efectuado sobre o nível de circuito, uma temática ainda pouco desenvolvida mas com um potencial imensurável.

É importante não esquecer que apesar do aeroporto internacional de Lisboa não pertencer ao conjunto de aeroportos mundiais com um maior volume de tráfego anualmente, o mesmo representa as portas de entrada para a Europa por via aérea do continente americano e africano, sendo precisamente estes dois continentes aqueles que se encontram, actualmente, numa fase de crescimento e com um maior interesse em se deslocarem para o mercado chinês.

Para além da sua localização privilegiada é importante não esquecer o forte empenho governamental para o desenvolvimento turístico da principal metrópole portuguesa assim como a crescente importância da mesma para o Mundo dos negócios, correlacionando-se com as conclusões obtidas no modelo no que diz respeito às variáveis dummy.

\section{Referências}

Bedo, D. e Dentinho, T. (2007) Avaliação dos destinos turísticos das ilhas dos Açores com base em modelos gravitacionais. Revista Portuguesa de Estudos Regionais, n. 14, pp. 35-52.

Borenstein, S. (1989) Hubs and high fares: dominance and market power in the U.S. airline industry. The RAND Journal of Economics, vol. 20, n. 3, pp. 344-365.

Condé, M. (2011) Estudo e previsão da demanda aeroportuária para a cidade do Rio de Janeiro. Journal of Transport Literature, vol. 5, n. 1, pp. 161-183.

Costa, J., Santos, L. e Yamashita, Y. (2008) Vocação turística das cidades brasileiras: análise de modelos de previsão de demanda do transporte aéreo. Simpósio de Transporte Aéreo - SITRAER, vol. 5, n. 1, pp. 209-219.

De La Mata, T., Lesage, J. e Llano, C. (2009) Social networks and trade of services: modeling interregional tourism flows with spatial and network autocorrelation effects. World Conference of Spatial Econometrics. 3. Barcelona papers. Barcelona: AQRIREA.

Forsyth, P. (2005) Tourism benefits and aviation policy. Martin kunz memorial lecture. Hamburg Aviation Conference, 16-18 Fevereiro 2005, pp. 1-20.

Fourie, J. e Santana-Gallego, M. (2011) The determinants of African tourism. Economic Research Southern Africa, n. 260, pp. 1-19.

Grampella, M., Martini, G., Scotti, D., Tassan, F. e Zambon, G. (2013) The environmental costs of airports' aviation activities: a panel data econometric analysis of Italian airports. Air Transport Research Society. Bergamo, Italy, 26-29 Junho 2013, pp. 1-31. 
Grimme, W. (2011) The growth of Arabian airlines from a german perspective - a study of the impacts of new air services to Asia. Journal of Air Transport Management, vol. 17, n. 6, pp. 333-338.

Grosche, T., Rothlauf, F. e Heinzl, A. (2007) Gravity models for airline passenger volume estimation. Jounal of Air Transport Management, vol. 13, n. 4, pp. 175-183.

Khadaroo, J. e Seetatanah, B. (2007) Transport infrastructure and tourism development. Annals of Tourism Research, vol. 34, n. 4, pp. 1021-1032.

Lesage, J. e Pace, R (2008) Spatial econometric modeling of origin-destination flows. Journal of Regional Science, vol. 48, n. 5, pp. 941-967.

Mukkala, K. e Tervo, H. (2012) Regional airports and regional growth in europe: which way does the causality run? RSA European Conference. Delf, Netherlands, 13-16 Maio 2012, pp. 1-19.

Pearce, D. (2003) Geografia do turismo: fluxos e regiões no mercado de viagens. São Paulo: Aleph.

Piane, G. e Kume, H. (2000) Fluxos bilaterais de comércio e blocos regionais: Uma aplicação do modelo gravitacional. Rio de Janeiro: Ipea.

Rocha, C. (2011) Ensaios sobre a demanda do transporte aéreo regional. Journal of Transport Literature, vol. 4, n. 1, pp.114-133.

Santos Silva, J. e Tenreyro, S. (2006) The log of gravity. The review of economics and statistics. vol. 88 , n. 4.

Schmidheiny, K. (2012) Panel data: fixed and random effects. Shot guides to microeconometrics. Unversität Basel.

Sem, A. e Smith, T. (1995) Gravity models of spatial interaction behaviour. Berlim: Springer.

Washington, S., Karlaftis, M. e Mannering, F. (2003) Statistical and econometric methods for transportation data analysis. Florida: CRC Press LLC.

Whalen, T. (2005) A panel data analysis of code sharing, antitrust immunity and open skies treaties in international aviation markets. Review of industrial organization, vol. 30, n. 1, pp. 39-61.

Wooldridge, J. (2002) Econometric analysis of cross section and panel data. Massachusetts: MIT Press.

Wooldridge, J. (2005) Introductory econometrics: a modern approach. $3^{\text {rd }}$ Edition. South-Wester: Cengage Learning.

Wu, S. (2012) The "Southwest effect" revisited: an empirical analysis of the effects of Southwest Airlines and JetBlue Airways on incumbent airlines from 1993 to 2009. The Michigan Journal of Business, vol. 5, n. 2, pp. 11-42. 